\title{
EDITORIAL
}

\section{Where next for lung cancer gene expression profiling?}

\author{
S.M. Janes and F. McCaughan
}

D iological understanding of lung cancer lags behind that of other tumours. The reasons for this are both biological and social. Lung cancer is largely derived from exposure to carcinogens in tobacco smoke. This leaves lung epithelium with a multitude of somatic chromosomal mutations, deletions and amplifications, and hence a multitude of possible target genes to study and understand. This is before the different cell types resident within the lung and the subsequent cancer subgroups are considered [1]. Unfortunately, research into this disease is also grossly under funded; and with the social vilification of smokers and accepting nihilism of doctors and patients alike, things are slow to change. However, up to $20 \%$ of lung cancers worldwide are in nonsmokers, and with an increasing proportion of lung cancer occurring in exsmokers, lobby groups are strengthening.

It is a huge challenge with the disrupted cancer genome to disentangle the critical or "driver" somatic changes in a cancer cell from the bystander changes of little biological impact. One way this has been addressed is by using modern array technology to examine the cancer genome (DNA) or transcriptome (mRNA). Such studies aim to define common signatures shared between tumours of the same type. It can be inferred that consistent significant biological changes within a specific tumour type are of functional significance and may represent suitable targets for therapeutic manipulation. Another goal of array-based data is to distinguish tumours of varying prognosis and ultimately to use this information to guide therapy.

In the current issue of the European Respiratory Journal, LARSEN et al. [2] have used gene expression array technology to understand why cancers of a similar stage appear to behave so differently. Their focus is on N1 disease, which in the current tumour, node, metastasis (TNM) staging system includes both metastases to ipsilateral, peribronchial, hilar and intrapulmonary nodes and the same nodes involved by direct extension of the tumour. Using a "genomic" approach LARSEN et al. [2] demonstrate that squamous cell carcinomas (SCCs) that metastasise to local nodes are clearly distinct from N1 nodes involved by direct extension at the mRNA transcript level. Furthermore, nodes involved by direct extension had a profile similar to N0 tumours. Survival data mirrored this finding, showing that metastatic N1 disease confers a worse survival than N0 disease, and while the numbers were too low to be

Centre for Respiratory Research, University College London, London, UK.

STATEMENT OF INTEREST: None declared.

CORRESPONDENCE: S.M. Janes, Centre for Respiratory Research, Rayne Building, University College London, London WC1E 6JJ, UK. Fax: 44 2076796973. E-mail: s.janes@ucl.ac.uk conclusive, N1 disease by direct invasion tends towards N0 disease rather than metastatic N1 disease. Not unreasonably, LARSEN et al. [2] conclude that N1 disease as a result of direct invasion behaves clinically and biologically as N0 disease and suggest that, if borne out in other cohorts, a re-evaluation of the staging and management of $\mathrm{N} 1$ disease would be appropriate. This in turn may reduce the number of patients subjected to adjuvant chemotherapy, and its associated risks and costs, without significant chance of success.

The study examines 59 lung cancers but only eight are in the group of nodal disease by direct invasion, so we should remain cautious. However, previous clinical studies do support the survival difference between the various N1 diseases [3, 4]. So should these findings be pursued, as LARSEN et al. [2] suggest, with new cohorts of patients? Perhaps. What the authors find with their sophisticated tool is interesting, but surely most physicians and cancer biologists would have predicted the outcome. The real interest of further studies and cohorts has to lie in the association of different genes with metastasis. Disseminated metastases remain the primary cause of mortality in all cancer patients [1-3]. It is critical that, as well as discriminating between tumours to inform either prognosis or treatment decisions, we also use these biological signatures to gain knowledge of the basic biology of lung cancer, which in turn will allow more effective treatment strategies to be devised.

Studies in breast cancer show that dissecting out a metastatic signature is worthwhile and can reveal molecular targets that could be attacked in order to stop metastasis. One such study, using a well-defined metastatic breast cancer human cell line xenografted onto immunodeficient mice, has identified a set of genes that mark and mediate breast cancer metastasis to the lungs [5]. The molecular requirements for metastasis must include tumour intravasation, survival in the circulation, extravasation into a second organ (this may be tissue specific), and finally growth and angiogenesis. Parent breast cancer cells were compared with cells that had metastasised to the lung and had subsequently been expanded and shown to be highly metastatic [5]. Transcriptomic microarray analysis revealed 95 genes that had a three-fold change in expression between the groups. The key to the success of studies such as these, however, is in the subsequent dissection of the data and challenging it in biological models. This takes a great deal of expertise and funding. Of these 95 genes, nine were chosen and it was largely found that when these genes individually were over-expressed, effects on metastasis formation were modest. However, consistent with the hypothesis that successful metastasis needs to overcome multiple barriers, it was found that over-expressing combinations of these genes led invariably to more aggressive metastatic activity. Therefore, 
these genes were indeed not only markers but also functional mediators of metastasis.

This leads to new avenues of research therapeutics. The same group recently established that four of the genes from the dataset facilitated new blood vessel formation, cell release into the circulation and pulmonary seeding [6]. The four genes examined were epidermal growth factor receptor (EGFR) ligand epiregulin, the cyclooxygenase COX2 and the matrix metalloproteinases 1 and 2. Subsequent individual targeting of these genes failed to prevent these tumour activities; however, their combined inhibition led to a profound reduction in each of the steps of metastatic progression. This included inhibition of EGFR and COX2. Importantly, these genes regulate not only metastasis but also primary tumour growth, hence supplying welcome new avenues for clinical research.

Other studies of lung cancer have established that gene expression profiles recapitulate histopathological classification [7] and predict survival [7-10]. LARSEN et al. [11] have also published an expression profiling paper revealing a 111-gene signature in SCCs that predicted recurrence and survival and was superior to TNM staging. Similarly, a study by POTTI et al. [12] established a gene expression profile for nonsmall cell carcinomas (they termed the lung metagene model) that predicted recurrence for individual patients better than clinical prognostic factors. The conclusions of РОТт et al. [12] were that it was possible to predict a subgroup of stage IA patients with poor prognosis that may have improved survival with adjuvant chemotherapy. A further study published in 2007 has proposed using a real-time reverse-transcriptase PCR approach, dubbed a five-gene signature, to stratify patients with respect to survival and relapse-free survival [13]. Although attractive in its simplicity, a number of reservations about the design of the study have been expressed [14].

SPIRA et al. [15] have asked a new question of similar techniques. Gene expression arrays were used, not to stratify patients into treatment or prognostic groups but with the aim of improving the early diagnosis of lung cancer. This is a key goal, as the main contributing factor to high mortality in lung cancer is its late stage at presentation. Bronchial brushings from histologically normal bronchial epithelium in the main bronchi were analysed and the expression signatures were used to correctly predict which patients did or did not have lung cancer, irrespective of the site or tissue type [15]. It may be that a similar strategy could be extended to exfoliated epithelial cells in sputum.

Therefore, there is an abundance of activity with respect to gene expression profiling and lung cancer. Although there is undoubted potential for the clinic, it is important to temper enthusiasm until there is a clear benefit to patient care, and we remain far from that point. It is important to be clear that there are, as yet, no randomised controlled trial data to support the use of expression profiling to guide decision making in lung cancer management.

Lung cancer research, as outlined previously in breast cancer research, needs to focus on dissecting out the molecular events underlying the critical steps in pathogenesis: the evolution of dysplastic epithelium, invasion and metastasis. Gene expression studies are one way in which the key driver genes for these steps may be identified. It is critical that clinical datasets are designed and used with this in mind, and that sufficient funding is allocated to carry out the basic science to further investigate those genes and pathways identified. The clues are there... let's watch this space.

\section{REFERENCES}

1 Giangreco A, Groot KR, Janes SM. Lung cancer and lung stem cells: strange bedfellows? Am J Respir Crit Care Med 2007; 175: 547-553.

2 Larsen JE, Pavey SJ, Bowman R, et al. Gene expression of lung squamous cell carcinoma reflects mode of lymph node involvement. Eur Respir J 2007; 30: 21-25.

3 van Velzen E, Snijder RJ, Brutel de la Riviere A, Elbers HJ, van den Bosch JM. Type of lymph node involvement influences survival rates in T1N1M0 non-small cell lung carcinoma. Lymph node involvement by direct extension compared with lobar and hilar node metastases. Chest 1996; 110: 1469-1473.

4 Marra A, Hillejan L, Zaboura G, Fujimoto T, Greschuchna D, Stamatis G. Pathologic N1 non-small cell lung cancer: correlation between pattern of lymphatic spread and prognosis. J Thorac Cardiovasc Surg 2003; 125: 543-553.

5 Minn AJ, Gupta GP, Siegel PM, et al. Genes that mediate breast cancer metastasis to lung. Nature 2005; 436: 518-524.

6 Gupta GP, Nguyen DX, Chiang AC, et al. Mediators of vascular remodelling co-opted for sequential steps in lung metastasis. Nature 2007; 446: 765-770.

7 Garber ME, Troyanskaya OG, Schluens K, et al. Diversity of gene expression in adenocarcinoma of the lung. Proc Natl Acad Sci USA 2001; 98: 13784-13789.

8 Bhattacharjee A, Richards WG, Staunton J, et al. Classification of human lung carcinomas by mRNA expression profiling reveals distinct adenocarcinoma subclasses. Proc Natl Acad Sci USA 2001; 98: 13790-13795.

9 Beer DG, Kardia SL, Huang CC, et al. Gene-expression profiles predict survival of patients with lung adenocarcinoma. Nat Med 2002; 8: 816-824.

10 Raponi M, Zhang Y, Yu J, et al. Gene expression signatures for predicting prognosis of squamous cell and adenocarcinomas of the lung. Cancer Res 2006; 66: 7466-7472.

11 Larsen JE, Pavey SJ, Passmore LH, et al. Expression profiling defines a recurrence signature in lung squamous cell carcinoma. Carcinogenesis 2007; 28: 760-766.

12 Potti A, Mukherjee S, Petersen R, et al. A genomic strategy to refine prognosis in early-stage non-small-cell lung cancer. N Engl J Med 2006; 355: 570-580.

13 Chen HY, Yu SL, Chen $\mathrm{CH}$, et al. A five-gene signature and clinical outcome in non-small-cell lung cancer. $N$ Engl J Med 2007; 356: 11-20.

14 Michiels S, Hill C. Five-gene signature in non-small-cell lung cancer. N Engl J Med 2007; 356: 1581-1582.

15 Spira A, Beane JE, Shah V, et al. Airway epithelial gene expression in the diagnostic evaluation of smokers with suspect lung cancer. Nat Med 2007; 13: 361-366. 